\title{
A white dwarf bound to the transiting planetary system WASP-98
}

\author{
John Southworth ${ }^{\oplus},{ }^{1 \star}$ Pier-Emmanuel Tremblay, ${ }^{2,3}$ Boris T. Gänsicke ${ }^{\oplus, 2,3}$ Daniel Evans ${ }^{1}$ \\ and Teo Močnik ${ }^{4}$ \\ ${ }^{1}$ Astrophysics Group, Keele University, Staffordshire ST5 5BG, UK \\ ${ }^{2}$ Department of Physics, University of Warwick, Coventry CV4 7AL, UK \\ ${ }^{3}$ Centre for Exoplanets and Habitability, University of Warwick, Coventry CV4 7AL, UK \\ ${ }^{4}$ Gemini Observatory, Northern Operations Centre, 670 N. A'ohoku Place, Hilo, HI 96720, USA
}

Accepted 2020 August 3. Received 2020 July 16; in original form 2020 March 17

\begin{abstract}
WASP-98 is a planetary system containing a hot Jupiter transiting a late-G dwarf. A fainter star, 12 arcsec away, has previously been identified as a white dwarf, with a distance and proper motion consistent with a physical association with the planetary system. We present spectroscopy of the white dwarf, with the aim of determining its mass, radius, and temperature and hence the age of the system. However, the spectra show the featureless continuum and lack of spectral lines characteristic of the DC class of white dwarfs. We therefore fitted theoretical white dwarf spectra to the ugriz apparent magnitudes and Gaia DR2 parallax of this object in order to determine its physical properties and the age of the system. We find that the system is old, with a lower limit of 3.6 Gyr, but theoretical uncertainties preclude a precise determination of its age. Its kinematics are consistent with membership of the thick disc, but do not allow us to rule out the thin-disc alternative. The old age and low metallicity of the system suggest that it is subject to an age-metallicity relation, but analysis of the most metal-rich and metal-poor transiting planetary systems yields only insubstantial evidence of this. We conclude that the study of bound white dwarfs can yield independent ages to planetary systems, but such analysis may be better suited to DA and DB rather than DC white dwarfs.
\end{abstract}

Key words: stars: fundamental parameters - stars: individual: WASP-98 - planetary systems - white dwarfs.

\section{INTRODUCTION}

The multiplicity of stars hosting transiting planets is an important piece in the jigsaw of our understanding of these systems. The majority of solar-type stars are members of binary systems (Duquennoy \& Mayor 1991; Raghavan et al. 2010; Duchêne \& Kraus 2013) and this is likely to have a significant effect on the incidence and properties of planets orbiting solar-type stars. Several studies have suggested that the formation of planets is affected by the binarity of the host star (Desidera \& Barbieri 2007): either hindered (Fragner, Nelson \& Kley 2011; Roell et al. 2012; Wang et al. 2015; Ziegler et al. 2020) or helped (Zhang et al. 2018; Ziegler et al. 2020), depending on the orbital separation of the two stars. Some observational studies have found a lower binary fraction for stars hosting planets versus those without planets (Roell et al. 2012; Kraus et al. 2016), whereas others have found an enhanced binary fraction (Ngo et al. 2016). Moe \& Kratter (2019) found that planet formation was unaffected by companions more distant than approximately 200 au. Once formed, the presence of the second star is expected to affect the orbital migration of the planet (Wu \& Murray 2003; Fabrycky \& Tremaine 2007) in a way that might be observationally testable (Morton \& Johnson 2011). These studies typically refer to hot Jupiters, which almost certainly form at relatively large distances from the host star and then migrate in (e.g. Ida \& Lin 2004); the prevalence of lower mass planets may be affected by binarity in different ways.

^E-mail: astro.js@keele.ac.uk
Of lesser scientific interest, but vital for correct determination of the physical properties of transiting extrasolar planets, is the effect of light from a nearby star on measurements of the physical properties of the system. This applies both if the nearby star is bound or merely an asterism, and the contamination can bias observationally derived quantities (e.g. Daemgen et al. 2009; Buchhave et al. 2011). Contaminating light acts to decrease the depth of the transit in light curves, leading to an underestimate of the radius of the planet. Analogously, stationary spectral lines from a nearby star will bias the measured orbital motion of the planet host star to lower values, so the planet appears to be less massive. These effects are undetectable from a transit light curve alone (Southworth 2009), unless the contamination is the dominant light source in the system (Bognár et al. 2015; Southworth et al. 2020), but can be straightforwardly accounted for if the properties of the nearby star are known (e.g. Southworth et al. 2010; Buchhave et al. 2011; Southworth et al. 2020).

A particularly good example of the problems raised by the multiplicity of planet host stars is that of WASP-20, which was found to be two stars separated by 0.26 arcsec when the system was imaged at high resolution (Evans, Southworth \& Smalley 2016a). It remains unclear which star actually hosts the planet; its measured mass and radius differ by factors of 1.3 and 3.4, respectively, between the two scenarios. In both cases, the planet's measured mass and radius are significantly higher than those found without accounting for binarity (Anderson et al. 2015). It is important to remember that binarity not only complicates the characterization of planets, but also decreases the probability of the planet being detected in the first place, due to the dilution of the photometric and spectroscopic signals of its existence. 
A less-travelled avenue of research is the possibility of degenerate companions to planet host stars. A consistent result from stellar evolutionary theory is that more massive stars evolve more quickly, so a wide binary system with a planet transiting the less massive star will in many cases become a transiting planetary system accompanied by a distant white dwarf companion. The physical effects on the planet and its host star will be modest for wide binaries, for two reasons. First, common-envelope evolution triggered by the evolution of the originally more massive star will preferentially remove tight $(\lesssim 10 \mathrm{au}$; Willems \& Kolb 2004) binary systems from the sample (Paczyński 1971). Secondly, there is an observed deficiency of planets around stars in binaries with separations of less than 200 au (see above).

The minimum binary separation for interactions to be negligible is not precisely known but is likely somewhere in the region of $100 \mathrm{au}$. One example is the GJ 86 system, which consists of a K1.5 V star and a white dwarf with an angular separation of 1.72 arcsec and an orbital separation of at least 28 au (Farihi et al. 2013) for which it has been postulated that the formation of the white dwarf triggered chromospheric activity in the K-star (Fuhrmann et al. 2014). A similar example is the $\mathrm{K} 2 \mathrm{~V}$ star HD 8049, which has a white dwarf at an angular separation of 1.57 arcsec (projected separation $50 \mathrm{au}$ ), and a chromospheric activity and rotation speed larger than expected for its age (Zurlo et al. 2013). Jeffries \& Stevens (1996) found that wind accretion from an asymptotic giant branch (AGB) star could spin-up a low-mass companion for final orbital separations of up to $100 \mathrm{au}$.

The effect of the white dwarf on the characterization of the system will also be minimal: The degenerate object will be much fainter than the planet host star, so the transit depth will be negligibly affected, and the faintness and spectral characteristics of the white dwarf mean that it will not have a significant effect on the radial velocities measured for the host star. However, the existence of the white dwarf will have the advantage that the age of the system can be constrained from the evolution of the progenitor and the cooling time of the white dwarf (see e.g. Fouesneau et al. 2019). So the presence of the white dwarf causes no problems in characterization of the system, but could aid in constraining its age, something that can be difficult for planet host stars less massive than the Sun due to their long evolutionary time-scales, our imperfect understanding of their structure (Maxted, Serenelli \& Southworth 2015a,b), and the possibility of star-planet interactions affecting age indicators of the host star (Poppenhaeger \& Wolk 2014; Maggio et al. 2015).

In this work, we present the characterization of a white dwarf companion to the transiting planetary system WASP-98. We discuss this object and the WASP-98 system, present the observations and analysis, discuss the kinematics of the system, and then conclude.

\section{THE WASP-98 SYSTEM}

WASP-98 was found to be a $\mathrm{G}$ dwarf hosting a transiting hot Jupiter by the SuperWASP survey (Hellier et al. 2014). Although its properties are mostly typical for a transiting planetary system with a hot Jupiter, it stood out as having an unusually low metallicity of $\left[\frac{\mathrm{Fe}}{\mathrm{H}}\right]=-0.60 \pm 0.19$. This value was then the second-lowest measured for a transiting planet host star, after $\left[\frac{\mathrm{Fe}}{\mathrm{H}}\right]=-0.64 \pm 0.15$ for WASP-112 (Anderson et al. 2014).

The WASP-98 system was studied in more detail by Mancini et al. (2016), who obtained new high-resolution échelle spectroscopy plus high-precision photometry of two transits in four optical passbands. The spectroscopic analysis yielded a revised metallicity measurement of $\left[\frac{\mathrm{Fe}}{\mathrm{H}}\right]=-0.49 \pm 0.10$, still among the lowest known for a
Table 1. Basic information and references for the planet host star (WASP98 A) and faint co-moving companion (WASP-98 B). Masses have two uncertainties: The first error bar is the statistical error and the second is the systematic error from stellar models (unknown $\mathrm{H} / \mathrm{He}$ abundance in the case of the white dwarf).

\begin{tabular}{lccc}
\hline Quantity & WASP-98 A & WASP-98 B & References \\
\hline Gaia DR2 ID & 4859136199796131200 & 4859136195500112256 & 1 \\
RA (J2000) & 035342.9627 & 035342.2632 & 1 \\
Dec. (J2000) & -341941.778 & -341950.415 & 1 \\
$G$ magnitude & $12.8678 \pm 0.0002$ & $20.1575 \pm 0.0062$ & 1 \\
$G_{\text {BP magnitude }}$ & $13.2447 \pm 0.0013$ & $19.940 \pm 0.087$ & 1 \\
$G_{\text {RP magnitude }}$ & $12.3370 \pm 0.0011$ & $19.779 \pm 0.126$ & 1 \\
Parallax (mas) & $3.520 \pm 0.020$ & $3.417 \pm 0.444$ & 1 \\
pmRA & $33.627 \pm 0.030$ & $33.381 \pm 0.738$ & 1 \\
(mas yr $^{-1}$ ) & & & \\
pmDec. & $-12.405 \pm 0.038$ & $-12.540 \pm 0.922$ & 1 \\
(mas yr ${ }^{-1}$ ) & & & \\
$V$ magnitude & $13.063 \pm 0.026$ & 20.18 & 2,3 \\
$J$ magnitude & $11.691 \pm 0.027$ & - & 4 \\
$H$ magnitude & $11.295 \pm 0.021$ & - & 4 \\
$K_{s}$ magnitude & $11.284 \pm 0.023$ & - & 4 \\
ATLAS $u$ & $14.360 \pm 0.035$ & $20.051 \pm 0.050$ & 5 \\
ATLAS $g$ & $13.246 \pm 0.013$ & $20.089 \pm 0.015$ & 5 \\
ATLAS $r$ & $12.807 \pm 0.013$ & $20.067 \pm 0.023$ & 5 \\
ATLAS $i$ & $12.641 \pm 0.012$ & $20.174 \pm 0.036$ & 5 \\
ATLAS $z$ & $12.539 \pm 0.055$ & $20.233 \pm 0.149$ & 5 \\
$T_{\text {eff }}(\mathrm{K})$ & $5473 \pm 121$ & $8270 \pm 210$ & 6,7 \\
Mass $\left(\mathrm{M}_{\odot}\right.$ ) & $0.809 \pm 0.053 \pm 0.036$ & $0.49 \pm 0.02 \pm 0.05$ & 6,7 \\
\hline
\end{tabular}

References: (1) Gaia DR2, pmRA, and pmDec. are the proper motions in RA and Dec., respectively; (2) APASS9 (Henden et al. 2012); (3) Gaia DR2 Documentation Release 1.1, section 5.3.7; (4) Cutri et al. (2003); (5) Shanks et al. (2015) including the nightly zero-point accuracy; (6) Mancini et al. (2016); (7) This work.

transiting planet host star. ${ }^{1}$ For reference, the physical properties returned by the analysis of Mancini et al. (2016) are a mass and a radius of $0.81 \mathrm{M}_{\odot}$ and $0.74 \mathrm{R}_{\odot}$ for the star, and $0.92 \mathrm{M}_{\text {Jup }}$ and $1.14 \mathrm{R}_{\mathrm{Jup}}$ for the planet.

Evans et al. (2016b) obtained high-resolution imaging of the sky area surrounding WASP-98 as part of the HITEP lucky-imaging survey. They found one faint point source, separated from WASP98 A by 12 arcsec. It had a bluer colour than the planet host star blue enough to be outside the range of validity of their $T_{\text {eff }}$ calibration - in the two non-standard passbands of the Two Colour Imager used (Skottfelt et al. 2015), indicative of a $T_{\text {eff }}$ in the region of $10000 \mathrm{~K}$. No further observations were obtained in the course of the project (Evans et al. 2018).

The publication of Data Release 2 (DR2) from the Gaia satellite (Gaia Collaboration et al. 2016, 2018) permitted the status of nearby stars to the HITEP targets to be examined in more detail (Evans 2018). We found the faint nearby object (hereafter named WASP$98 \mathrm{~B})$ to have a parallax and proper motion consistent with that of the planet host star (WASP-98 A), suggesting that the two systems were bound and, thus, share a common evolutionary history. Basic information on both objects is contained in Table 1. Based on their sky positions in Gaia DR2, the angular separation of the two objects is $12.23 \mathrm{arcsec}$ and the corresponding minimum linear separation is 3500 au.

The properties of WASP-98 B - intrinsically faint yet blue in optical colour - are consistent with it being a white dwarf. This is supported by Gentile Fusillo et al. (2019) who constructed a

\footnotetext{
${ }^{1}$ The metallicity of WASP-98 A, measured by Mancini et al. (2016), is the ninth lowest in the TEPCat catalogue of transiting planetary systems (Southworth 2011). The catalogue was accessed on 2020/03/03 at URL http://www.astro.keele.ac.uk/jkt/tepcat/
} 


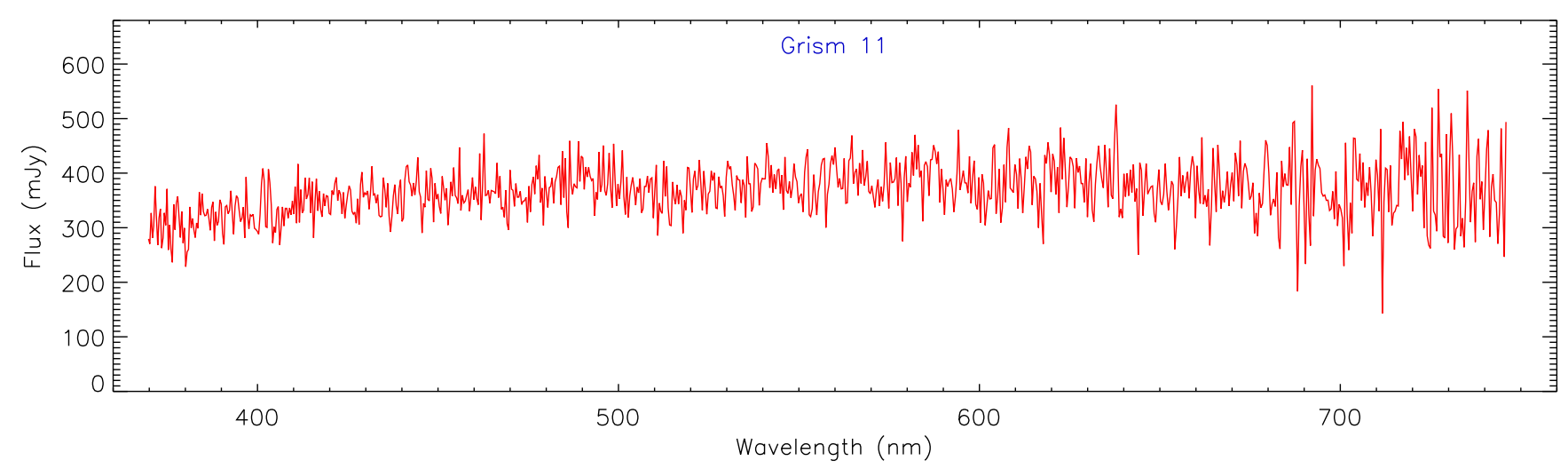

Figure 1. NTT/EFOSC2 spectrum of WASP-98 B obtained using grism 11. The spectrum shown is the mean of three individual spectra after flux calibration using the standard star LTT 1788.

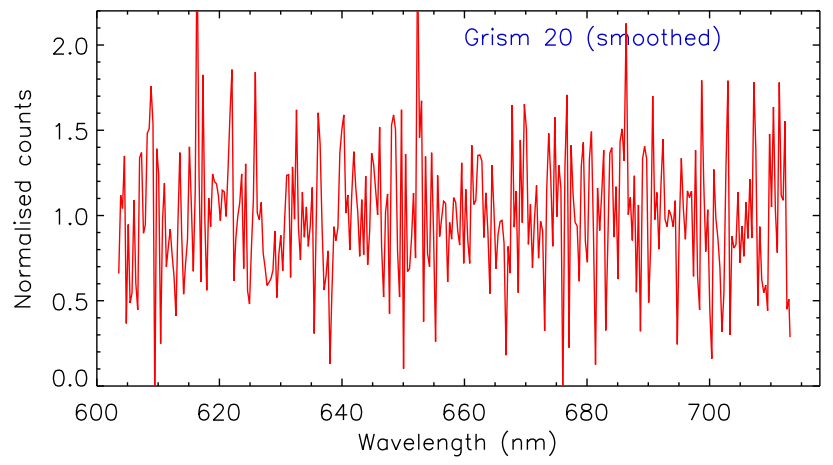

Figure 2. NTT/EFOSC2 spectrum of WASP-98 B obtained using grism 20. The spectrum has been rectified to a continuum level of unity using a straightline fit. Each group of three consecutive data points has been averaged in order to reduce the noise level for visual inspection.

catalogue of white dwarfs based on Gaia DR2 data. They found the object (labelled WD J035342.22-341950.22) to have a $T_{\text {eff }}$ of approximately $10000 \mathrm{~K}$, with a probability of 0.995 of being a white dwarf. Based on this supposition, we decided to obtain follow-up spectroscopy of WASP-98 B, with the aim of determining its physical properties and, thus, the age of the whole system.

\section{OBSERVATIONS}

Various surveys, most notably Gaia DR2 and the ESO ATLAS Public Survey, provide measured apparent magnitudes for both WASP-98 A and WASP-98 B (Table 1). WASP-98 A is well-characterized from studies of the planet transits (Hellier et al. 2014; Mancini et al. 2016), but little was known about WASP-98 B. We therefore obtained follow-up spectroscopy of this object.

\subsection{NTT spectroscopy}

We used the ESO New Technology Telescope (NTT) with the EFOSC2 spectrograph (Buzzoni et al. 1984) to obtain spectroscopy of WASP-98 B covering three wavelength intervals. Three consecutive spectra were taken on the night of 2018 July 9, with exposure times of $1800 \mathrm{~s}$ each, using grism 11 to give a wavelength coverage of $370-746 \mathrm{~nm}$ at a mean reciprocal dispersion of $0.42 \mathrm{~nm}$ pixel $^{-1}$ (Fig. 1). One $1800 \mathrm{~s}$ spectrum was obtained on 2018 July 8 using grism 20 to cover the $\mathrm{H} \alpha$ line $(603-713 \mathrm{~nm})$ at higher resolution $\left(0.11 \mathrm{~nm}\right.$ pixel $\left.^{-1}\right)$ and is shown in Fig. 2. We also obtained a spectrum with grism 7 whilst cloud-dodging, but its signal-to-noise ratio $(\mathrm{S} / \mathrm{N})$ is too low to be useful. In all cases, we used a 1 arcsec slit, yielding resolutions of $1.6 \mathrm{~nm}$ for grism 11 and $0.4 \mathrm{~nm}$ for grism 20 .

The data were reduced using a pipeline under development by the first author. The steps included bias and flat-field calibration, cosmic ray rejection (van Dokkum 2001), aperture extraction, wavelength calibration using a helium-argon emission lamp, and flux calibration using an observation of the standard star LTT 1788.

The spectrum from grism 11 shows a flat continuum with no evidence for either absorption or emission lines. The spectrum from grism 20 shows no clear $\mathrm{H} \alpha$ line absorption. This is surprising for a white dwarf, as such objects typically show strong Balmer absorption lines that would have been obvious in our spectra. The NTT spectra have a low $\mathrm{S} / \mathrm{N}$ due to the faintness of the star twinned with the modest aperture of the telescope.

\subsection{SALT spectroscopy}

In an attempt to characterize WASP-98 B via detection of weaker spectral lines that would not be apparent in the NTT spectra, we obtained a spectrum using the Southern African Large Telescope (SALT) and the Robert Stobie Spectrograph (RSS; Burgh et al. 2003) via a DDT application. The observations were obtained on the night of 2018 December 31 in service mode during dark time, and comprised two consecutive spectra each with an exposure time of $1460 \mathrm{~s}$. We used the PG900 grating with a camera tilt angle of $29^{\circ} .5$, yielding a spectrum covering $406-703 \mathrm{~nm}$ at a reciprocal dispersion of $0.09 \mathrm{~nm}$ pixel $^{-1}$. The spectrum is spread over three CCD detectors, so suffers from the presence of two gaps between the CCDs. The data were reduced as above, except that the wavelength calibration was obtained using an argon lamp and no flux calibration was performed.

The two spectra were combined and data affected by strong nightsky emission lines were masked. The spectra from the individual CCDs were then each normalized to a continuum level of unity by dividing by a quadratic function. The resulting spectrum (Fig. 3) shows no evidence for absorption lines from either hydrogen or helium, suggesting that the target star is a DC white dwarf.

\section{ANALYSIS OF THE WHITE DWARF}

Because the spectrum of WASP-98 B is featureless at the $\mathrm{S} / \mathrm{N}$ of our data, we have instead relied on survey photometry to determine its properties. The Gaia DR2 $G_{\mathrm{BP}}$ and $G_{\mathrm{RP}}$ magnitudes are unreliable 


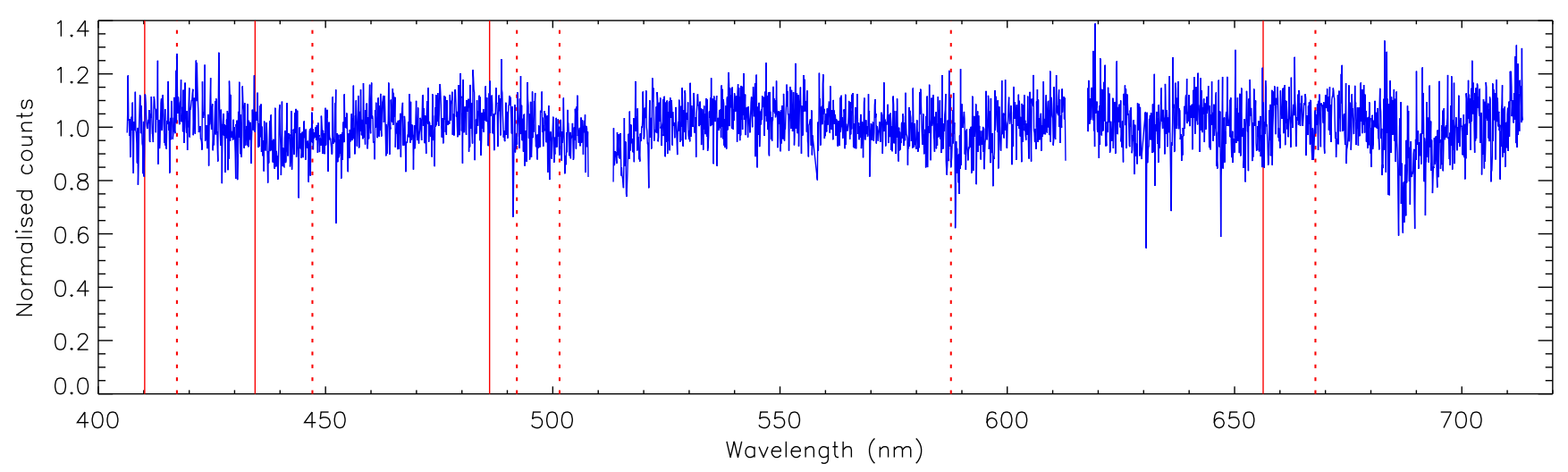

Figure 3. SALT/RSS spectrum of WASP-98 B. The spectrum has been rectified to a continuum level of unity by dividing by a fitted quadratic polynomial. The positions of the hydrogen Balmer lines are indicated using vertical solid lines. A selection of helium lines typically strong in DB atmospheres (Liebert 1977) have their positions shown using vertical dotted lines.

due to poor background subtraction. ${ }^{2}$ This is likely due to the presence of much brighter WASP-98 A at 12 arcsec, but may also be caused by WASP-98 B being close to the faint limit of Gaia. We therefore obtained ugriz magnitudes from the ATLAS survey (Shanks et al. 2015). The interstellar extinction between us and the WASP-98 system is very low at $0.008 \pm 0.005 \mathrm{mag}^{3}$ (Lallement et al. 2014, 2018). We fitted the dereddened photometry, but emphasize that the correction is below the statistical error bars.

We converted ATLAS AB magnitudes to the equivalent SDSS system following equation (1) of Gentile Fusillo et al. (2017) (also see Shanks et al. 2015). Gentile Fusillo et al. (2017) found that for a sample of overlapping ATLAS and SDSS point sources with $g<19.5$, mean differences between corrected ATLAS and SDSS magnitudes are smaller than combined statistical uncertainties. We therefore fitted the converted ATLAS photometry with our grid of model atmosphere fluxes integrated over nominal SDSS ugriz. passbands.

Gentile Fusillo et al. (2019) identified a bifurcation between hydrogen- and helium-atmosphere white dwarfs in the Gaia HR diagram for the range $0.0 \lesssim G_{\mathrm{BP}}-G_{\mathrm{RP}} \lesssim 0.7$ corresponding to $11000 \gtrsim T_{\text {eff }} \gtrsim 7000 \mathrm{~K}$. This bifurcation is poorly reproduced by evolution tracks assuming pure-helium model atmospheres for the helium-rich population. Bergeron et al. (2019) have shown that by relying on helium-rich atmospheres with trace amounts of hydrogen, they could provide a much better fit to the helium-rich white dwarf cooling sequence. From our observations of WASP-98 B the upper limit on $\mathrm{H} / \mathrm{He}$ is $\approx 10^{-3.5}$ in number of atoms (see also fig. 8 of Rolland, Bergeron \& Fontaine 2018). The immediate progenitors of DC white dwarfs are thought to be warmer DB and DBA stars with helium and hydrogen lines, for which the median $\mathrm{H} / \mathrm{He}$ value is $\approx 10^{-5}$ (Rolland et al. 2018). We therefore used this atmospheric composition as the most likely scenario for WASP-98 B.

Fig. 4 presents our best fit to the ATLAS photometry of WASP$98 \mathrm{~B}$ using mixed $\mathrm{H} / \mathrm{He}=10^{-5}$ model atmospheres and the massradius relation of Fontaine, Brassard \& Bergeron (2001) for thin hydrogen layers. We obtained $T_{\text {eff }}=8270 \pm 210 \mathrm{~K}, \log g=7.84 \pm 0.06$, and $M=0.49 \pm 0.02 \mathrm{M}_{\odot}$ with the error bars corresponding to

${ }^{2}$ The BP/RP excess factor (background subtraction parameter) is 1.499 , which is large according to section 5.5.2 of the Gaia DR2 documentation: https://gea.esac.esa.int/archive/documentation /GDR2/pdf/GaiaDR2_documentation_1.2.pdf.

${ }^{3}$ https://stilism.obspm.fr/

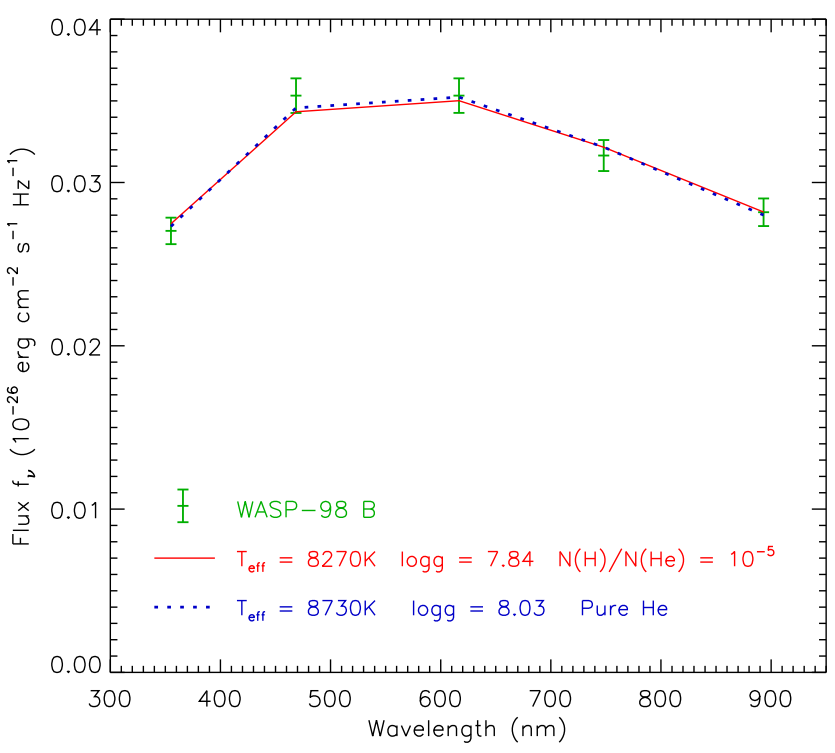

Figure 4. Fit of ATLAS ugriz photometry of WASP-98 B with theoretical white dwarf model atmospheres, one helium-only and one with $\mathrm{H} / \mathrm{He}=10^{-5}$ by number. The best-fitting atmospheric parameters are identified on the panel.

statistical uncertainties. Varying the fixed hydrogen abundance by \pm 1 dex does not significantly change our solution. However, purehelium model atmospheres lead to a much more significant shift to $T_{\text {eff }}=8730 \pm 220 \mathrm{~K}, \log g=8.03 \pm 0.07$, and $M=0.59 \pm 0.04 \mathrm{M}_{\odot}$.

The cooling age of the white dwarf is $0.83 \pm 0.09$ and $0.92 \pm 0.09 \mathrm{Gyr}$ with the mixed and pure-helium solutions, respectively (Fontaine et al. 2001). The mass of WASP-98 B is possibly significantly lower than the Gaia field white dwarf average mass of $0.58-0.59 \mathrm{M}_{\odot}$ (Tremblay et al. 2019), which suggests a mainsequence progenitor with a relatively long lifetime. The mixed $\mathrm{H} / \mathrm{He}$ solution for WASP-98 B is problematic as the derived white dwarf mass is smaller than those of old halo white dwarfs (Kalirai 2012), which represent a lower limit on the mass of the oldest white dwarfs produced through single star evolution in our Galaxy. In other words, we would infer a total age well above $\approx 12 \mathrm{Gyr}$, which has unlikely given the kinematics of WASP-98 B. It is more likely that our white dwarf mass is underestimated owing to systematic issues with the photometry or an incorrect assumption in the modelling. 


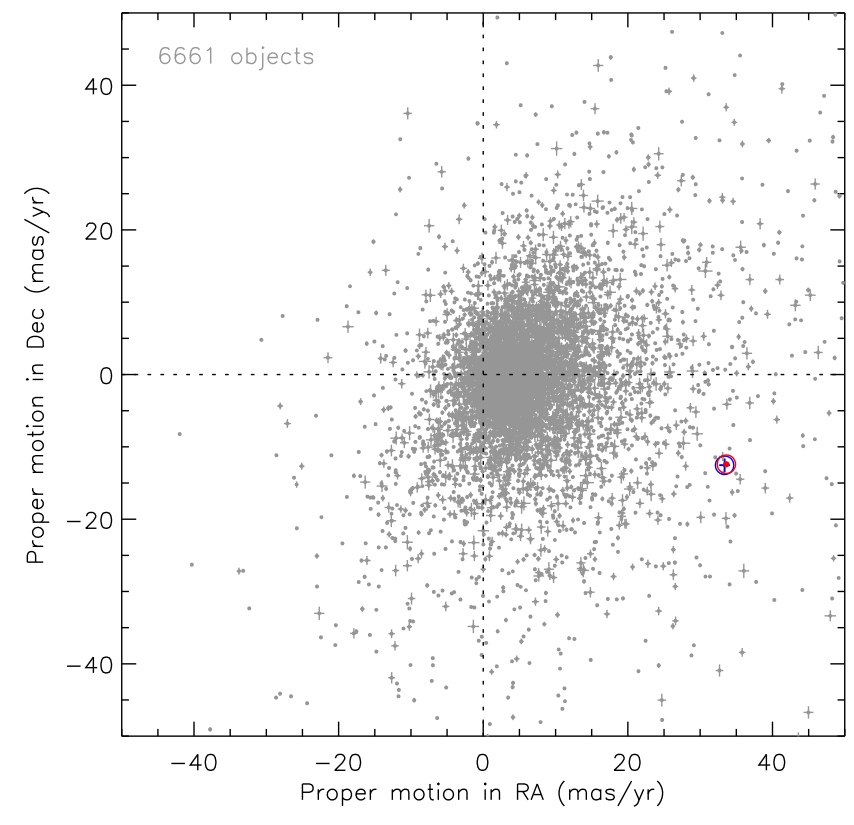

Figure 5. Plot of the proper motions in RA and Dec. of all objects in Gaia DR2 with measured proper motions and sky positions within $1^{\circ}$ of WASP98 A (grey points). WASP-98 A and WASP-98 B are shown using red and blue open circles, respectively, that are almost entirely overlapping.

We therefore included an additional systematic mass error of $0.05 \mathrm{M}_{\odot}$, to allow for a small hydrogen abundance (approximately $10^{-7}$ ), without including the unphysical case of a pure-helium composition as this is ruled out by Gaia DR2 for most DC white dwarfs. In this case, the lower limit to the age of the system becomes 3.6 Gyr, using the initial-to-final mass relation of Kalirai et al. (2008) and the main-sequence lifetime from Hurley, Pols \& Tout (2000). The initial mass of the white dwarf can be constrained to be between 0.80 (Kalirai 2012) and $1.53 \mathrm{M}_{\odot}$ (Kalirai et al. 2008) based on our lower and upper limits for the final white dwarf mass, respectively. The effect of the low metallicity of the system on its age and initial mass inferred from the initial-to-final mass relation is significantly below the uncertainties in these quantities (Kalirai et al. 2009; Cummings et al. 2018).

The ATLAS survey has a relatively small overlap with known spectroscopic white dwarf catalogues. Only a handful of ATLAS sources have been confirmed as DC white dwarfs from spectroscopy (e.g. from the Montreal White Dwarf Database; Dufour et al. 2017). It is also possible that the ATLAS photometry is contaminated by WASP-98 A. For these reasons, it is difficult to conclude whether WASP-98 B is truly overluminous, hence larger, less massive, and older than field white dwarfs of similar colours and spectral type. This leaves us with a poorly constrained total age for the system, but with an indication that the WASP-98 system may be older than the average field star.

\section{KINEMATICS OF THE WASP-98 SYSTEM}

There are two points to consider for the kinematics of the system: the relation between the white dwarf and the planet host star, and the galactic population they belong to. To address the first point, we obtained a catalogue of all detected sources within $1^{\circ}$ of the sky position of WASP-98 A from Gaia DR2 and plotted their proper motions in Fig. 5. The white dwarf and the planet host star are highlighted in this figure using blue and red points. It can be seen that the proper motions of the two objects are extremely similar, which supports their physical association. Their proper motions are also significantly offset from the bulk motion of the other stars in the plot, suggesting that they have a much higher space velocity than most other stars in that sky region.

We determined the galactic space velocity of the system using the proper motion and systemic velocity of the planet host star (Hellier et al. 2014; Gaia Collaboration et al. 2018) and the method of Johnson \& Soderblom (1987). ${ }^{4}$ We found $U=-17.9 \mathrm{~km} \mathrm{~s}^{-1}, V=$ $-5.5 \mathrm{~km} \mathrm{~s}^{-1}$ and $W=64.4 \mathrm{~km} \mathrm{~s}^{-1}$; these values have been corrected to the local standard of rest as found by Coșkunoğlu et al. (2011). We determined the probability that the system consists of thin or thick disc stars using the method of Bensby et al. (2005) and the approach of Coșkunoğlu et al. (2011), finding a 77 per cent probability that it is thick disc and a 23 per cent probability that it is thin disc. We are not able to definitively assign it to a single galactic population on this evidence alone.

Mackereth \& Bovy (2018) determined approximate values of the galactic orbital parameters of the 6.6 million stars with radial-velocity measurements in Gaia DR2. For WASP-98, they found a small orbital eccentricity of $0.097 \pm 0.002$ and a large maximum distance from the galactic plane of $1414 \pm 54 \mathrm{pc}$. The uncertainties in these parameters are underestimated as they do not include the dominant contribution from the choice of galactic potential (Mackereth \& Bovy 2018). This maximum distance is far greater than the scale height of the thin disc of our galaxy (approximately $300 \mathrm{pc}$ according to e.g. Gilmore \& Reid 1983), which suggests a thick-disc membership for WASP-98. If so, its age is likely to be old; for example, Fantin et al. (2019) found all thick-disc stars to have formed at $9.8 \pm 1.5 \mathrm{Gyr}$. This supports an age of roughly $10 \mathrm{Gyr}$ for the WASP-98 system, but is unfortunately not a conclusive evidence.

\section{SUMMARY AND DISCUSSION}

We have presented the characterization of a faint white dwarf companion, WASP-98 B, to a transiting planet host star, WASP98 A. WASP-98 B was originally discovered by our group and was independently found to be a white dwarf by Gentile Fusillo et al. (2019) based on its absolute magnitude and colours in the Gaia passbands. The distances and proper motions of the two objects in the Gaia DR2 catalogue agree, suggesting that they are physically related. The proper motion of the system is relatively large and together with the systemic velocity indicates (with a probability of 77 percent) that the system is part of the thick disc of the Milky Way. The sky-projected separation of the two resolved components is 12.23 arcsec, which corresponds to a minimum physical separation of $3500 \mathrm{au}$. The progenitor of the white dwarf had an initial mass greater than the current white dwarf mass, so the conservation of orbital energy requires the initial orbit to be smaller. The upper limit on the initial mass is $1.53 \mathrm{M}_{\odot}$ (Section 4), which corresponds to a lower limit on the initial orbital separation of $1350 \mathrm{au}$.

Our intention was to determine the mass, radius, and temperature of WASP-98 B in order to use it as an age estimator for the planetary system. We therefore obtained spectroscopy of this white dwarf using the NTT and SALT, but found no evidence for any spectral absorption or emission lines. WASP-98 B is therefore a DC white dwarf, whose properties had to be determined by fitting theoretical spectra to its ugriz magnitudes and the distance of the system from the Gaia DR2

\footnotetext{
${ }^{4}$ To determine the space motions, we used the GAL_UVW procedure available in the NASA ASTROLIB library of IDL routines.
} 
parallax of WASP-98 A. We found a good fit to these measurements for two different sets of theoretical spectra, differing in the amount of hydrogen adopted when constructing the model atmosphere. Both alternatives indicate that the progenitor was of relatively low mass, and thus the white dwarf is old. We place a lower limit on its age of $3.6 \mathrm{Gyr}$ and caution that the upper limit is well beyond the age of the Universe.

We conclude that the WASP-98 system is old, in agreement with its likely membership of the thick-disc population, a finding that is a useful addition to our understanding of the planetary component. Mancini et al. (2016) obtained and modelled highprecision photometry of two transits in the planetary system. The low mass of the star precluded a useful constraint on its age from its density (which is measured from the transit shape), which was found to be $2.7_{-2.5}^{+6.2}$ (stat. $)_{-1.0}^{+2.9}$ (sys.) Gyr. This work therefore provides the first informative constraint on the age of this system.

The old age and low metallicity of the WASP-98 system is interesting in the context of the age-metallicity relation in our Galaxy expected from the chemical evolution of stellar populations (Feltzing \& Chiba 2013). The reality of this relation is unclear, as it appears to exist in the thick-disc but not thin-disc populations (Casagrande et al. 2011; Bensby, Feltzing \& Oey 2014; Casagrande et al. 2016). Do the known transiting planet host stars exhibit an agemetallicity relation? To test this, we queried the TEPCat catalogue (Southworth 2011) for objects with $[\mathrm{Fe} / \mathrm{H}] \leq-0.4$ and $[\mathrm{Fe} / \mathrm{H}] \geq$ +0.4 , and then searched the literature for published age estimates for the systems identified. The low-metallicity sample contains 16 stars of which 15 have age estimates: the mean age is $7.7 \mathrm{Gyr}$, with a median of $10.0 \mathrm{Gyr}$, and a standard deviation of $3.7 \mathrm{Gyr}$. The highmetallicity sample contains 15 stars, of which 14 have age estimates: the mean age is $5.8 \mathrm{Gyr}$, with a median of $7.6 \mathrm{Gyr}$, and a standard deviation of 3.6 Gyr. The difference in ages between these two samples is not statistically significant and the ages themselves have been estimated in a wide variety of ways (including consideration of the $[\mathrm{Fe} / \mathrm{H}]$ values in some cases). A detailed analysis of a possible age-metallicity relation in planet host stars is advocated, based on homogeneous age estimates for the host stars. It would need to account for the different stellar mass distributions in the low- and high-metallicity samples, metallicity-dependent detection biases, the known bias towards higher metallicities for the host stars of giant planets (Gonzalez 1997; Valenti \& Fischer 2005; Wang \& Fischer 2015), and the enhancement of $\alpha$-elements in lower metallicity stars relative to the solar abundance pattern.

Whilst the current work was in progress, Mugrauer (2019) published a detailed analysis of 1367 extrasolar planet hosts within 500 pc. Using Gaia DR2, they detected faint companions to 204 planetary systems. Within this sample are eight white dwarf companions. WASP-98 B was included in this category based on its faintness, blue colour in optical photometry, and non-detection in 2MASS. We confirm this deduction. Finally, Bonavita \& Desidera (2020) recently performed a similar analysis on a sample of 850 stars with planets detected via precise radial-velocity measurements. They detected 10 white dwarf companions, although only 9 are mentioned in their notes on individual systems, of which 3 were previously unknown. These companions may be useful in constraining the ages of the planetary systems, especially if they show the strong absorption lines typical of DA and DB white dwarfs.

\section{ACKNOWLEDGEMENTS}

Based on observations made with ESO telescopes at the La Silla Paranal Observatories under programme ID 0101.C-0071 (PI: South- worth), on observations obtained with the Southern African Large Telescope (SALT) under program 2018-2-DDT-002 (PI: Southworth), and on data products from observations made with ESO Telescopes at the La Silla Paranal Observatory under program ID 177.A-3011. We thank Luigi Mancini for helpful discussions, and an anonymous referee whose report led to a significant improvement in the quality of the manuscript. The research leading to these results has received funding from the European Research Council under the European Union's Horizon 2020 research and innovation programme no. 677706 (WD3D). The following internet-based resources were used in research for this paper: the ESO Digitized Sky Survey; the NASA Astrophysics Data System; the SIMBAD database operated at CDS, Strasbourg, France; and the ar $\chi$ iv scientific paper preprint service operated by Cornell University.

\section{DATA AVAILABILITY}

The raw data underlying this article are available in the ESO Archive by searching for programme ID 0101.C-0071 or by sky position, and in the SALT Archive by searching for programme ID 20182-DDT-002 or by sky position. The reduced spectra will be made available at the CDS (http: //cdsweb.u-strasbg.fr/) and http://www.astro.keele.ac.uk/jkt/ and are available on reasonable request to the corresponding author.

\section{REFERENCES}

Anderson D. R. et al., 2014, MNRAS, preprint (arXiv:1410.3449)

Anderson D. R. et al., 2015, A\&A, 575, A61

Bensby T., Feltzing S., Lundström I., Ilyin I., 2005, A\&A, 433, 185

Bensby T., Feltzing S., Oey M. S., 2014, A\&A, 562, A71

Bergeron P., Dufour P., Fontaine G., Coutu S., Blouin S., Genest-Beaulieu C., Bédard A., Rolland B., 2019, ApJ, 876, 67

Bognár Z. et al., 2015, A\&A, 581, A77

Bonavita M., Desidera S., 2020, Galaxies, 8, 16

Buchhave L. A. et al., 2011, ApJS, 197, 3

Burgh E. B., Nordsieck K. H., Kobulnicky H. A., Williams T. B., O’Donoghue D., Smith M. P., Percival J. W., 2003, in Iye M., Moorwood A. F. M., eds, Proc. Conf. Ser. SPIE Vol. 4841, Instrument Design and Performance for Optical/Infrared Ground-Based Telescopes. SPIE, Bellingham, p. 1463

Buzzoni B. et al., 1984, The Messenger, 38, 9

Casagrande L. et al., 2016, MNRAS, 455, 987

Casagrande L., Schönrich R., Asplund M., Cassisi S., Ramírez I., Meléndez J., Bensby T., Feltzing S., 2011, A\&A, 530, A138

Coșkunoğlu B. et al., 2011, MNRAS, 412, 1237

Cummings J. D., Kalirai J. S., Tremblay P. E., Ramirez-Ruiz E., Choi J., 2018, ApJ, 866, 21

Cutri R. M. et al., 2003, 2MASS All Sky Catalogue of Point Sources, NASA/IPAC Infrared Sci. Arch., Caltech, US

Daemgen S., Hormuth F., Brandner W., Bergfors C., Janson M., Hippler S., Henning T., 2009, A\&A, 498, 567

Desidera S., Barbieri M., 2007, A\&A, 462, 345

Duchêne G., Kraus A., 2013, ARA\&A, 51, 269

Dufour P., Blouin S., Coutu S., Fortin-Archambault M., Thibeault C., Bergeron P., Fontaine G., 2017, Tremblay E., Gänsicke B., Marsh T., eds, ASP Conf. Ser. Vol. 509, The Montreal White Dwarf Database: a Tool for the Community. Astron. Soc. Pac., San Francisco, p. 3

Duquennoy A., Mayor M., 1991, A\&A, 248, 485

Evans D. F., 2018, Res. Notes Am. Astron. Soc., 2, 20

Evans D. F., Southworth J., Smalley B., 2016a, ApJ, 833, L19

Evans D. F. et al., 2016b, A\&A, 589, A58

Evans D. F. et al., 2018, A\&A, 610, A20

Fabrycky D., Tremaine S., 2007, ApJ, 669, 1298

Fantin N. J. et al., 2019, ApJ, 887, 148 
Farihi J., Bond H. E., Dufour P., Haghighipour N., Schaefer G. H., Holberg J. B., Barstow M. A., Burleigh M. R., 2013, MNRAS, 430, 652

Feltzing S., Chiba M., 2013, New Ann. Rev., 57, 80

Fontaine G., Brassard P., Bergeron P., 2001, PASP, 113, 409

Fouesneau M., Rix H.-W., von Hippel T., Hogg D. W., Tian H., 2019, ApJ, 870,9

Fragner M. M., Nelson R. P., Kley W., 2011, A\&A, 528, A40

Fuhrmann K., Chini R., Buda L. S., Pozo Nuñez F., 2014, ApJ, 785, 68

Gaia Collaboration et al., 2016, A\&A, 595, 36

Gaia Collaboration et al., 2018, A\&A, 616, 22

Gentile Fusillo N. P. et al., 2017, MNRAS, 469, 621

Gentile Fusillo N. P. et al., 2019, MNRAS, 482, 4570

Gilmore G., Reid N., 1983, MNRAS, 202, 1025

Gonzalez G., 1997, MNRAS, 285, 403

Hellier C. et al., 2014, MNRAS, 440, 1982

Henden A. A., Levine S. E., Terrell D., Smith T. C., Welch D., 2012, J. Am. Assoc. Var. Star Obs., 40, 430

Hurley J. R., Pols O. R., Tout C. A., 2000, MNRAS, 315, 543

Ida S., Lin D. N. C., 2004, ApJ, 604, 388

Jeffries R. D., Stevens I. R., 1996, MNRAS, 279, 180

Johnson D. R. H., Soderblom D. R., 1987, AJ, 93, 864

Kalirai J. S., 2012, Nature, 486, 90

Kalirai J. S., Hansen B. M. S., Kelson D. D., Reitzel D. B., Rich R. M., Richer H. B., 2008, ApJ, 676, 594

Kalirai J. S., Saul Davis D., Richer H. B., Bergeron P., Catelan M., Hansen B. M. S., Rich R. M., 2009, ApJ, 705, 408

Kraus A. L., Ireland M. J., Huber D., Mann A. W., Dupuy T. J., 2016, AJ, 152,8

Lallement R., Vergely J. L., Valette B., Puspitarini L., Eyer L., Casagrande L., 2014, A\&A, 561, A91

Lallement R. et al., 2018, A\&A, 616, A132

Liebert J., 1977, ApJ, 214, 446

Mackereth J. T., Bovy J., 2018, PASP, 130, 114501

Maggio A. et al., 2015, ApJ, 811, L2

Mancini L., Giordano M., Mollière P., Southworth J., Brahm R., Ciceri S., Henning T., 2016, MNRAS, 461, 1053
Maxted P. F. L., Serenelli A. M., Southworth J., 2015a, A\&A, 575, A36

Maxted P. F. L., Serenelli A. M., Southworth J., 2015b, A\&A, 577, A90

Moe M., Kratter K. M., 2019, MNRAS, preprint (arXiv:1912.01699)

Morton T. D., Johnson J. A., 2011, ApJ, 729, 138

Mugrauer M., 2019, MNRAS, 490, 5088

Ngo H. et al., 2016, ApJ, 827, 8

Paczyński B., 1971, ARA\&A, 9, 183

Poppenhaeger K., Wolk S. J., 2014, A\&A, 565, L1

Raghavan D. et al., 2010, ApJS, 190, 1

Roell T., Neuhäuser R., Seifahrt A., Mugrauer M., 2012, A\&A, 542, A92

Rolland B., Bergeron P., Fontaine G., 2018, ApJ, 857, 56

Shanks T. et al., 2015, MNRAS, 451, 4238

Skottfelt J. et al., 2015, A\&A, 574, 54

Southworth J., 2009, MNRAS, 394, 272

Southworth J., 2011, MNRAS, 417, 2166

Southworth J. et al., 2010, MNRAS, 408, 1680

Southworth J., Bohn A. J., Kenworthy M. A., Ginski C., Mancini L., 2020, A\&A, 635, A74

Tremblay P. E., Cukanovaite E., Gentile Fusillo N. P., Cunningham T., Hollands M. A., 2019, MNRAS, 482, 5222

Valenti J. A., Fischer D. A., 2005, ApJS, 159, 141

van Dokkum P. G., 2001, PASP, 113, 1420

Wang J., Fischer D. A., 2015, AJ, 149, 14

Wang J., Fischer D. A., Horch E. P., Xie J.-W., 2015, ApJ, 806, 248

Willems B., Kolb U., 2004, A\&A, 419, 1057

Wu Y., Murray N., 2003, ApJ, 589, 605

Zhang Y., Li Q., Xie J.-W., Zhou J.-L., Liu H.-G., Zhang H., 2018, ApJ, 861, 116

Ziegler C., Tokovinin A., Briceno C., Mang J., Law N., Mann A. W., 2020, AJ, 159, 19

Zurlo A. et al., 2013, A\&A, 554, A21

This paper has been typeset from a $\mathrm{T}_{\mathrm{E}} \mathrm{X} / \mathrm{L} \mathrm{T}_{\mathrm{E}} \mathrm{X}$ file prepared by the author. 